María Fernanda Argandoña-Mendoza; Carlos Enrique García-Vera; Patricio Alfredo Vallejo-Valdivieso http://dx.doi.org/10.35381/e.k.v3i6.855

\title{
Flipped classroom y Educación para el emprendimiento durante la pandemia por COVID-19
}

Flipped classroom and entrepreneurship Education during pandemic COVID-19

\author{
María Fernanda Argandoña-Mendoza \\ margandona8801@pucem.edu.ec \\ Pontificia Universidad Católica del Ecuador, Extensión Manabí, Portoviejo \\ Ecuador \\ https://orcid.org/0000-0001-7322-4222 \\ Carlos Enrique García-Vera \\ cgarcia@pucem.edu.ec \\ Pontificia Universidad Católica del Ecuador, Extensión Manabí, Portoviejo \\ Ecuador \\ https://orcid.org/0000-0003-1743-7682 \\ Patricio Alfredo Vallejo-Valdivieso \\ pvallejo@pucem.edu.ec \\ patricio2871@yahoo.es \\ Pontificia Universidad Católica del Ecuador, Extensión Manabí, Portoviejo \\ Ecuador \\ https://orcid.org/0000-0003-3248-7864
}

Recepción: 10 abril 2020

Revisado: 15 de mayo 2020

Aprobación: 30 junio 2020

Publicación: 23 de julio 2020 


\title{
RESUMEN
}

El objetivo se centró en analizar el flipped classroom y la educación para el emprendimiento durante pandemia por COVID-19 en contexto del Ecuador. Se desarrolló desde el método analítico, mediante un tipo de investigación descriptiva documental con diseño bibliográfico. Las categorías centrales del flipped classroom, permiten sintetizar que la mayor responsabilidad de generación del aprendizaje se focaliza en el estudiante, siendo el rol del docente el de asesor en procura de encaminar al estudiante a la construcción de su conocimiento. Las categorías centrales del emprendimiento educativo, develan primordialmente que la tendencia curricular se focaliza en lo holístico y multidisciplinar. El flipped classroom y el emprendimiento educativo se relacionan en cuanto tienen como eje fundamental el aprendizaje activo con la finalidad de conocer el contexto de actuación, mediante la investigación y generación de competencias - tanto habilidades cognitivas y no cognitivas.

Descriptores: Aprendizaje activo; método de aprendizaje; educación y empleo; pertinencia de la educación. (Palabras tomadas del Tesauro UNESCO).

\begin{abstract}
The objective is based upon analyzing the flipped classroom and entrepreneurship education during the COVID-19 pandemic in the context of Ecuador. It was developed from the analytical method, through a type of descriptive documentary research with a bibliographic design. The central categories of the flipped classroom allows summarizing that the main responsibility for generating learning is focused on the students, with the role of the teacher as an advisor in order to direct them to the construction of their knowledge. The central categories of educational entrepreneurship reveal primarily that the curricular trend focalizes on a holistic and a multidisciplinary approach. The flipped classroom and educational entrepreneurship are related since they hold active learning as a fundamental axis in order to know the context of action, through research and generation of competences - considering both non-cognitive and cognitive skills.
\end{abstract}

Descriptors: Active learning; learning methods; education and employment; educational relevance. (Words taken from the UNESCO Thesaurus). 
María Fernanda Argandoña-Mendoza; Carlos Enrique García-Vera; Patricio Alfredo Vallejo-Valdivieso

\section{INTRODUCCIÓN}

La educación históricamente ha estado ligada a la formación de las personas con fines de prepararlas para asumir roles en la sociedad, uno de ellos, el acceso al trabajo o al empleo, para esto se ha diseñado un sistema educativo de menor a mayor complejidad, basado en diversas modalidades, enfoques, doctrinas, por las cuales el currículo educativo se orienta en la concesión de programar las metas a alcanzar por parte de los estudiantes (Córica\& Otero, 2014).

En Latinoamérica la brecha laboral se distancia entre la población, hasta el punto que en ciertos países se han adoptado políticas para el primer empleo juvenil o recién egresado universitario, como una modalidad que busca garantizar la experiencia mínima exigida por las empresas, con lo cual, se genera mayor competitividad por una plaza laboral (Miranda \& Alfredo, 2018). En el fondo esta modalidad refuerza el modelo asalariado, el cual se ve trastocado cada día a causa de la globalidad empresarial. Esto, aunado a la actual crisis generada por la pandemia por COVID-19, en donde se ha evidenciado no solo la pérdida de plazas de trabajo; sino, que también ha develando la fragilidad de los sistemas económicos, (Pierre-Álvarez \& Harris, 2020), advierten los retos a los cuales se enfrenta la región en base a transcender la problemática sanitaria, económica, pero también educativa; pues, como expresan (Argandoña-Mendoza, et al., 2020), la educación ha girado vertiginosamente en su accionar, resquebrajándose la planificación curricular pensada desde el modelo presencial.

Desde lo planteado se observa la perspectiva socio económica y educativa del Ecuador, siendo pertinente innovar educativamente en función de proponer alternativas viables para desarrollar un modelo educativo concomitante a la productividad nacional, teniendo como alternativa, retomar el planteamiento de la educación para el emprendimiento, por cuanto contribuye a la conformación de un actor social en función de formarse para no solo ser empleado, sino, además para concebir empleos. Siendo recurrente en una sociedad digital, tecnológica, donde se percibe un mayor éxito en los negocios fundados en la internet. De allí que (Contreras-Velásquez, et al., 2017), 
María Fernanda Argandoña-Mendoza; Carlos Enrique García-Vera; Patricio Alfredo Vallejo-Valdivieso

indican la necesidad de afrontar un modelo educativo interdisciplinario de emprendimiento, enfoque relacionado a formar al estudiante desde diversas competencias con la finalidad de estar en concordancia con la realidad global.

En correspondencia a lo planteado, (Sánchez-García, et al., 2017), expresan que el emprendimiento ha contribuido al desarrollo de países denominados tercermundistas, sin embargo en Latinoamérica se debe profundizar para consolidar tal concepción, sobre todo en el ámbito educativo - académico desde donde se debe promover una renovación curricular, así como la concepción pedagógica por parte del docente, siendo esto indispensable para conformar un modelo en repuestas a las brechas socioeconómicas que se han profundizado a causa del COVID-19.

En este sentido, (Osorio-Tinoco \& Pereira-Laverde, 2011), plantean la necesidad de formar desde una óptica basada en educar no sobre el emprendimiento, sino, para el emprendimiento, lo cual implica transcender lo teórico para complementarlo con la práctica. El aprender haciendo se suma como posible contribuyente para lograra la articulación de una visión emprendedora en los estudiantes, lo contrario implica negar la posibilidad de sentar las bases formativas para un real emprendimiento.

Para ello, es necesario la conformación de un liderazgo en el estudiante que contribuya a afrontar retos para emprender en función de las múltiples necesidades existentes (Berkovich \& Eyal, 2015), así como tener clara la concepción hacia donde debe enfocar el proyecto de vida como presupuesto que contribuye a fijar metas claras, siendo pertinente fomentar la inteligencia emocional para tal fin, por cuanto permite elevar la estima en función de asumir riesgos productivos (García-Cabrera, et al., 2016).

Por otro lado, (Osorio \& Londoño-Roldán, 2016), declaran que la intención de emprendimiento en estudiantes de educación media de modo efectivo, radica en si perciben como proyecto de vida ser empresarios; sin embargo, el fondo del emprendimiento educativo no solo debe limitarse a una concepción netamente empresarial, sino, la de formar para un liderazgo proactivo que progresivamente contribuya al estudiante a descubrir capacidades y habilidades en medio de las 


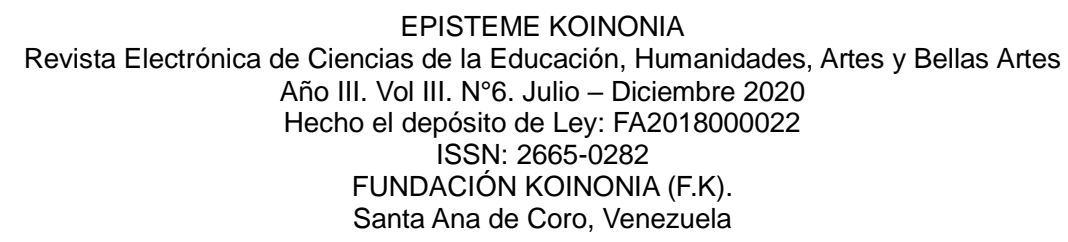

María Fernanda Argandoña-Mendoza; Carlos Enrique García-Vera; Patricio Alfredo Vallejo-Valdivieso adversidades, limitaciones, siendo posible trabajar en la optimización de los recursos disponibles, situación que permitirá comprender la importancia de transcender a nuevos modelos socio económicos en función de contar con diversas posibilidades de crecer en una sociedad competitiva y basada cada día en el desarrollo económico como supremacía moral.

En este contexto, es indispensable promover en la educación, la espiritualidad como eje integrador de la ética, que es dimensión fundamental del ser humano para constituir una persona integral desde el rol laboral que desempeñe, así la educación emprendedora tiende a formar desde una concepción humanista como centro del desarrollo humano (Palacio-Vargas, 2015). Por otro lado, (Oliver, et al., 2016), plantean la necesidad de generar la promoción del emprendimiento en los estudiantes como opción de crecimiento socio económico, además de cultural-tecnológico, sobre todo si se proyecta un emprendimiento global desde la internet, siendo necesario manejar idiomas y herramientas informáticas para interconectarse al mundo, lo cual demanda de la educación un giro en su accionar transmisionista al de generador de aprendizajes.

Para forjar tal concepción, se requiere adoptar el enfoque centrado en el estudiante, en donde este toma el protagonismo de su aprendizaje, mediante un proceso de búsqueda, indagación, innovación, confirmación de la información obtenida, con apoyo de las TIC, siendo pertinente en tal sentido y en concordancia con la actual situación educativa por COVID-19, tener en miramiento el Flipped Classroom como método de aprendizaje, que además es conocido como aula invertida (Gilboy, et al., 2015).

El Flipped Classroom contribuye a promover competencias afines al emprendimiento, por cuanto el estudiante requiere trazar un plan de trabajo para lograr las metas establecidas en la planificación curricular; además es, a partir de orientaciones o asesoramientos por parte del docente, quien desarrolla una serie de actividades demostrativas del aprendizaje alcanzado, el cual se caracteriza por ser duradero en el tiempo (Mero-Chávez, et al., 2019). 
En cierto modo el Flipped Classroom para ser efectivo, necesita que el estudiante sea proactivo para generar aprendizaje, siendo esto un factor fundamental del emprendimiento, aunado a la indagación de información, lo cual se puede relacionar con el estudio de mercado y la factibilidad de encontrar el nicho empresarial donde asentar su emprendimiento, desarrollándose un proceso de metacognición en favor de construir conocimiento para el mejor provecho del discente (Galindo-Domínguez, 2018). En correspondencia con lo abordado, el actual papel de trabajo se circunscribe a la tipología de revisión, por lo que su objetivo se centra en analizar el flipped classroom y la educación para el emprendimiento durante la pandemia por COVID-19 en el contexto del Ecuador.

\section{MÉTODO}

El trabajo se desarrolló desde el método analítico, mediante un tipo de investigación descriptiva documental con diseño bibliográfico, empleándose el análisis de contenido como técnica para la revisión de artículos publicados en revistas arbitradas en referencia a las variables flipped classroom y emprendimiento educativo.

\section{ANÁLISIS Y DISCUSIÓN DE RESULTADOS}

\section{Flipped classroom}

En la revisión analítica realizada por (Låg \& Saele, 2019) en bases de datos de primer orden editorial y en lengua no hispana, verifican que el flipped classroom tiene un favorable efecto estadístico sobre la educación tradicional, por cuanto los estudiantes consideran que se sienten con mayor motivación al aprendizaje, desde la perspectiva de planificar, indagar, buscar, organizar, la información para ser sistematizada en presentaciones en el aula de clases o en el entorno virtual, con la finalidad de construir el aprendizaje desde una concepción cooperativa y colaborativa con sus compañeros y docentes. 
En este contexto, el cooperativo y colaborativo se potencia en la medida que el flipped classroom, se asocia con rasgos de la personalidad como la amabilidad, afabilidad, caracterizadas por el disfrute del estudiante al participar en la clase (Lyons, et al., 2017), planteándose además que los estudiantes con rasgos de personalidad conflictiva, presentan inconvenientes para integrarse activamente en los subprocesos desarrollados a través del flipped classroom o aula invertida.

Así mismo, el rol de asesor del docente contribuye a generar auto reflexión en el estudiante con la intención de descubrir sus potencialidades, debilidades, desde una evaluación integral de sí mismo, incluyendo lo emocional, lo cual complementa la posibilidad de lograr la autonomía hacia el aprendizaje, siendo necesario el debido acompañamiento del docente, así como del entorno familiar, brindando la confianza necesaria al estudiante para alcanzar un favorable rendimiento académico a partir del develamiento de sus fortalezas (Yoon, et al., 2018).

Lo planteado hasta ahora, permite develar la importancia que juega la inteligencia emocional en el desarrollo efectivo del flipped classroom, siendo pertinente que el estudiante sea apoyado para descubrir su potencial emocional (Pulido-Acosta \& Herrera-Clavero, 2019)., así como docentes y directivos deben diseñar planes de intervención educativa mediante la aplicación de la inteligencia emocional, lo cual coadyuva en constituir un liderazgo democrático donde el estudiante se identifica con el rol de asesor del docente (Chen\&Guo, 2020), siendo percibido favorable por parte de los docentes, quienes consideran la inteligencia emocional como un factor fundamental para el aprendizaje (Cejudo \& López-Delgado, 2017).

En cuanto a la evaluación mediante el flipped classroom, el estudio de (Samuel, 2019), revela que los estudiantes valoraron en mayor porcentaje al docente cuando aplicó evaluaciones basadas en el método del aula invertida, por cuanto la grabación de las clases, videos y otros recursos audiovisuales, contribuyen a una percepción favorable del aprendizaje, teniéndose en cuenta que la evaluación debe ser centrada desde el enfoque constructivista, lo cual requiere la demostración de las habilidades y 
María Fernanda Argandoña-Mendoza; Carlos Enrique García-Vera; Patricio Alfredo Vallejo-Valdivieso competencias que el estudiante ha adquirido (Vidal-Ledo, et al., 2016).

Con respecto al grado de complejidad del aprendizaje de las matemáticas, el estudio de (Madrid-García, et al., 2018), evidencia que no existieron diferencias significativas entre el grupo experimental y control en donde se aplicó el flipped classroom para el aprendizaje de ecuaciones matemáticas, por lo que es necesario considerar la preparación previa de docentes y estudiantes, con la finalidad de asumirlo con mayor efectividad.

La efectividad del flipped classroom se relaciona al hecho de adecuarse al enfoque centrado en el estudiante (Peche-Cruz \& Giraldo-Supo, 2019), siendo pertinente considerar que esta perspectiva requiere una interacción entre docente y estudiante en adhesión a establecer una relación pedagógica basada en la cooperación para lograr las metas propuestas, y en donde la investigación se centra en facilitar la acción educativa, propiciándose con ello un proceso metacognitivo al transformar la información inicial en aprendizaje significativo (Muñoz-Morales, et al., 2019), ante lo cual, el acompañamiento familiar se hace indispensable en los primeros pasos del estudiante en el aula invertida (Páez, 2018). 


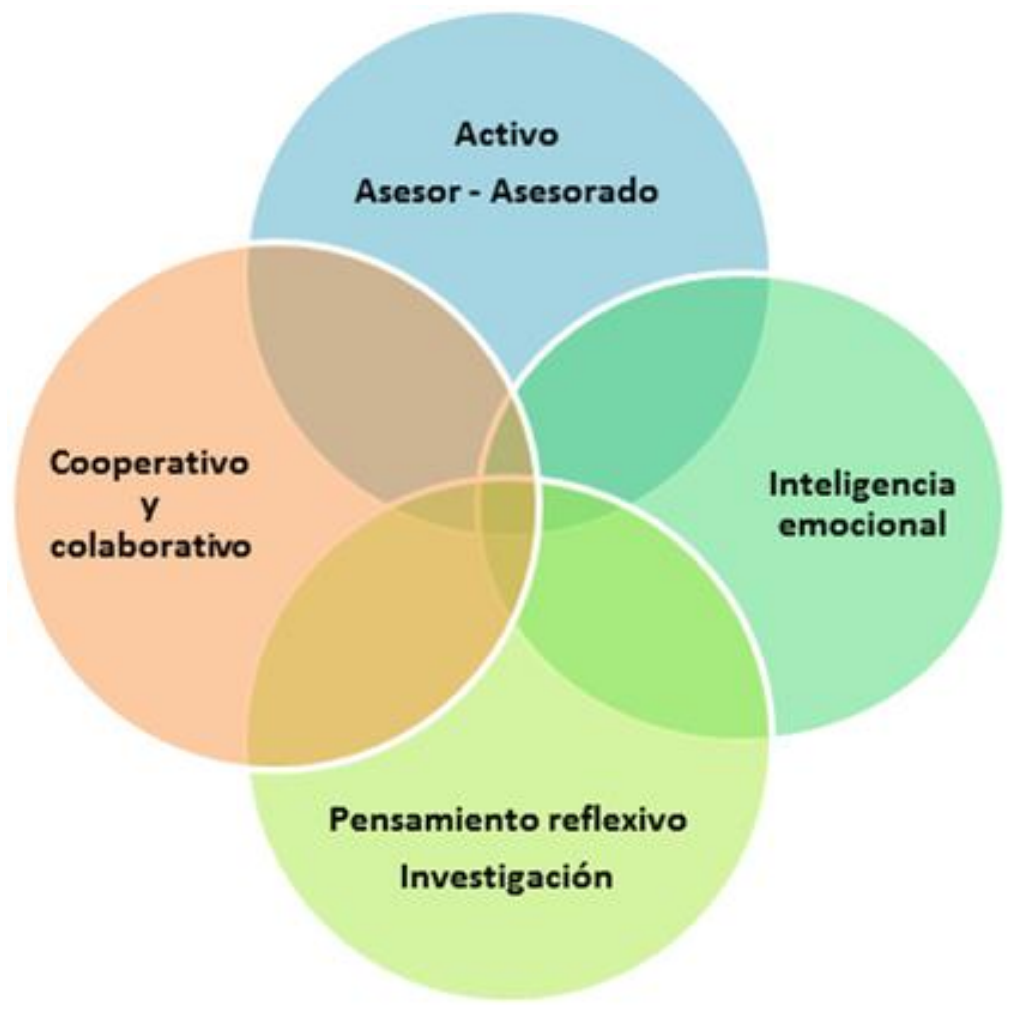

Figura 1. Categorías centrales del flipped classroom.

Fuente: A partir de información analizada (2020)

Las categorías centrales del flipped classroom, permiten sintetizar que la mayor responsabilidad de generación del aprendizaje se focaliza en el estudiante, siendo el rol del docente el de asesor en procura de encaminar al estudiante a la construcción de su conocimiento, mediante la búsqueda de información de primera fuente; para lo cual es indispensable desarrollar el pensamiento reflexivo desde la investigación, así el discente no estará conforme con lo abordado en el aula de clases, sino, que se propondrá indagar más sobre un determinado tema, hasta comprenderlo y usar en la vida cotidiana.

Para esto es necesario que el docente, familia, estudiantes, conformen equipos de 
trabajos basados en la investigación, sustentando desde la visión cooperativa y colaborativa del aprendizaje, con la finalidad de sumar esfuerzos para superar las debilidades de los participantes, promoviéndose la sinergia en función de lograr metas comunes, siendo necesario además aplicar la inteligencia emocional, la cual permite el desarrollo de relaciones intra e interpersonales afables entre los miembros del equipo. De esta manera se promueve una visión educativa proactiva para articular acciones favorables al logro del crecimiento integral del discente.

\section{Emprendimiento educativo}

El emprendimiento en los últimos años ha tenido en la educación un aliado para formar desde una perspectiva de negocios, siendo recurrente lo relacionado al contexto digital, pues la internet ha permitido el diseño de un sector empresarial que hace 30 años atrás era inimaginable. Esto, aunado a las crisis sociales y económicas que han obligado a emprender para superarlas, mas el enfoque de Estado - Nación como ente burocrático para el sostenimiento de los salarios, que parece agotarse cada día, contribuyen al fortalecimiento del tema emprendimiento como opción de crecimiento socio económico. Sin embargo, (Davey, et al., 2016), advierten que en relación a las políticas para incentivar el emprendimiento, es escasa la literatura a nivel científico, lo que determina la necesidad de explorar en esta área desde el sustento investigativo. Así el sistema educativo puede concebir una oportunidad para regenerarse curricularmente con la intención de acoplarse a las necesidades de formación en emprendimiento, además de explotar un campo casi inexplorado desde el abordaje de la investigación.

En este sentido, el rol de las universidades para profesionalizar en contexto al emprendimiento, es un factor determinante para crear una generación de profesionales basada en la óptica de emprender, así como desde un enfoque holístico. De allí que los estudios de posgrados deben caracterizarse por ser un espacio para la conformación del emprendimiento en razón de brindar respuestas multidisciplinares a los diversos problemas socio económicos, así se pasa de un modelo racionalista a uno en donde el 


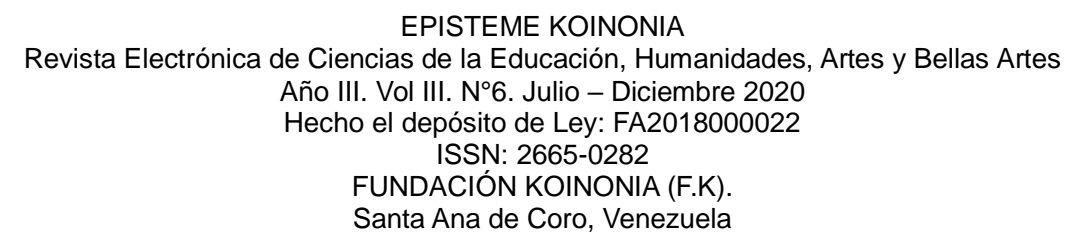

María Fernanda Argandoña-Mendoza; Carlos Enrique García-Vera; Patricio Alfredo Vallejo-Valdivieso pragmatismo educativo con visión proactiva, contribuye a fortalecer una nueva visión profesional de la economía colectiva.

La visión multidisciplinar del emprendimiento educativo, inserta lo relacionado a las artes visuales promocionado desde la universidad lo referente a emprender en un campo similar al mercado tecnológico por cuanto la empresa audiovisual artística es una de las fuentes económicas de mayor producción (Damásio, \&Bicacro, 2017), así se promueve en las universidades la transcendencia del modelo clásico de las escuelas de negocio al de emprendimiento desde una concepción multidisciplinar (Kazakeviciute, et al., 2016), así el currículo se diseña en función de promover el pensamiento interdisciplinario, para que el emprendedor puede captar la realidad desde lo sistémico de la realidad.

La formación holística del emprendimiento se basa en el aprendizaje por proyectos, el cual contribuye en fomentar competencias y habilidades no cognitivas en el estudiante en proyección del éxito profesional (Rodríguez \& Lieber, 2020), en este sentido el aprendizaje afectivo como estrategia para promover lo emocional es un factor esencial para suscitar el pensamiento emprendedor en los estudiantes (llonen \& Heinonen, 2018). Es por ello que (Bagiatis, et al., 2020), indican que el emprendimiento debe promoverse en la educación formal, mediante un currículo flexible que permita comprender la importancia del emprendedor como factor primordial en el crecimiento económico de la sociedad.

Un factor importante de la educación emprendedora, es la de enseñar a asumir riesgos mediante el trabajo en equipo, propiciándose con ello un esfuerzo cooperativo en superar las debilidades en función del bien común (Arpiainen\&Kurczewska, 2017), y promoviéndose habilidades para ejercer la ciudadanía como factor de desempeño ético de la persona; siendo fundamental incentivar estas habilidades cognitivas, emocionales y procedimentales en la educación primaria, con la intención de propiciar desde temprana edad, la visión emprendedora (Hassi, 2016). 


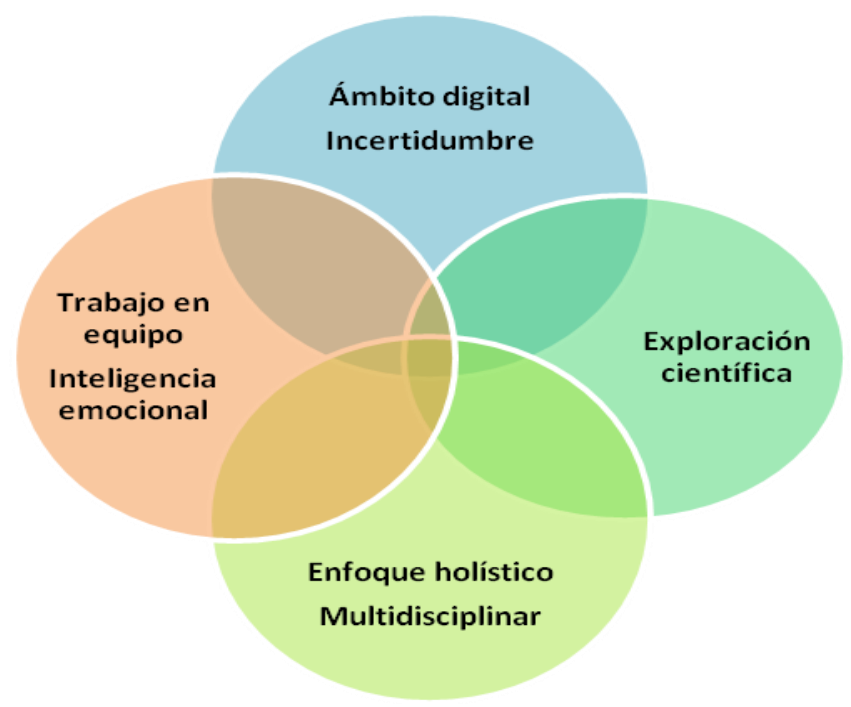

Figura 2. Categorías centrales del emprendimiento educativo Fuente: A partir de información analizada (2020)

Las categorías centrales del emprendimiento educativo, develan primordialmente que la tendencia curricular se focaliza desde un enfoque holístico multidisciplinar, lo cual implica formar al emprendedor para enfrentarse a un mundo basado en lo digital, pero sobre todo en la incertidumbre, lo que quiere decir que no se tiene certeza del mañana, por cuanto la sociedad global es dinámica y busca copiar el modelo de actualización de lo informático-tecnológico. En consecuencia, debe prepararse integralmente el emprendedor por cuanto esto le permitirá tener las competencias y habilidades cognitivas y no cognitivas para asumir con efectividad el reto de emprender, siendo pertinente contar con lo emocional como factor equilibrador del actuar en función de promover el trabajo en equipo para tener mayor opción de transcender y permanecer en el mercado de forma efectiva. 
María Fernanda Argandoña-Mendoza; Carlos Enrique García-Vera; Patricio Alfredo Vallejo-Valdivieso

\section{Flipped classroom y educación para el emprendimiento durante pandemia por COVID-19 en contexto del Ecuador}

La pandemia por COVID-19 ha generado la incertidumbre de qué ocurrirá en el futuro y cómo será el desarrollo de los diversos procesos sociales, entre los cuales se encuentran la educación y la economía como principales en el desarrollo del país; sin embargo, el confinamiento ha conllevado al teletrabajo y a la teleeducación a dimensiones, aunque conocidas, no habituales en la práctica social ecuatoriana. Resultando con esto un cambio paradigmático, el cual ha sido asumido sobre la marcha de los acontecimientos, descubriendo la necesidad de actualizar el currículo escolar, así como de generación de fuentes empleos para estabilizar el sector económico.

Lo planteado devela la propuesta de asumir desde el sistema educativo ecuatoriano, la educación basada en el emprendimiento; de ser así, se deberá generar una reestructuración curricular fundamentada desde el accionar holístico y el aprendizaje basado en flipped classroom, por cuanto contribuye a formar al estudiante en una concepción proactiva en búsqueda de la fuente informativa, estudiando el contexto, actuando emocionalmente de modo asertivo y propiciando un liderazgo sinérgico que incentive el trabajo cooperativo y colaborativo. De de ese modo, se concibe la posibilidad de contar con una generación de emprendedores dispuestos a asumir los retos venideros en función del dinamismo global desde el contexto educativo y económico, así se forjará una educación en pertinencia a la transformación social.

\section{CONCLUSIÓN}

El flipped classroom y el emprendimiento educativo se relacionan en cuanto tienen como eje fundamental el aprendizaje activo con la finalidad de conocer el contexto de actuación, mediante la investigación y la generación de competencias - habilidades no cognitivas y cognitivas, fundadas desde el trabajo en equipo cooperativo - colaborativo, y con una perspectiva del manejo asertivo de las emociones; lo que propicia un ciudadano proactivo en posibilidad de asumir retos para afrontar las necesidades 
económicas - tecnológicas del mundo global. Esto representa una oportunidad para el Ecuador de analizar su actualización educativa-económica en razón de gestar posibilidades de promover inversiones en favor del crecimiento integral de la sociedad, por lo que es necesario promover la formación permanente en los docentes y estudiantes en conformidad de comprender y actuar en base al enfoque de emprendimiento educativo.

\section{FINANCIAMIENTO}

No monetario

\section{AGRADECIMIENTOS}

A la Pontificia Universidad Católica del Ecuador, Extensión Manabí, Portoviejo, por su motivación y apoyo en el desarrollo de la investigación.

\section{REFERENCIAS}

Argandoña-Mendoza, M., García-Mejía, R., Ayón-Parrales, E., \& Zambrano-Zambrano, Y. (2020). Investigación e innovación educativa: Reto escolar por COVID-19 en el Ecuador. [Educational research and innovation: School challenge for COVID-19 in Ecuador]. EPISTEME KOINONIA, 3(5), 162-182. http://dx.doi.org/10.35381/e.k.v3i5.726

Arpiainen, R.-L., \&Kurczewska, A. (2017). Learning risk-taking and coping with uncertainty through experiential, team-based entrepreneurship education. Industry and Higher Education, 31(3), 143-155. https://doi.org/10.1177/0950422217700994

Bagiatis, C., Saiti, A., \&Chletsos, M. (2020). Entrepreneurship, economic crisis, and the role of higher education: Evidence from Greece. Industry and Higher Education, 34(3), 177-189. https://doi.org/10.1177/0950422219883832

Berkovich, I., \&Eyal, O. (2015). Educational Leaders and Emotions: An International Review of Empirical Evidence 1992-2012. Review of Educational Research, 85(1), 129-167. https://doi.org/10.3102/0034654314550046 
Cejudo, J, \&López-Delgado, M. (2017).Importancia de la inteligencia emocional en la práctica docente: un estudio con maestros. [Importance of emotional intelligence in the teaching practice: A study with primary education teachers]. Psicología Educativa, 23(1), 29-36. https://doi.org/10.1016/i.pse.2016.11.001

Córica, A \& Otero, A. (2014). Educación y empleo en América Latina: Entre tendencias y alcances. [Education and employment in Latin America: Between trends and scope]. Papeles de población, 20(82), 167-200.

Contreras-Velásquez, J, Wilches-Duran, S, Graterol-Rivas, M, \& Bautista-Sandoval, M. (2017). Educación Superior y la Formación en Emprendimiento Interdisciplinario: Un Caso de Estudio. [Higher Education and Training in Interdisciplinary Entrepreneurship: A Study Case]. Formación universitaria, 10(3), 1120. https://dx.doi.org/10.4067/S0718-50062017000300003

Chen, J., \&Guo, W. (2020). Emotional intelligence can make a difference: The impact of principals' emotional intelligence on teaching strategy mediated by instructional leadership. Educational Management Administration \& Leadership, 48(1), 82105. https://doi.org/10.1177/1741143218781066

Davey, T., Hannon, P., \&Penaluna, A. (2016). Entrepreneurship education and the role of universities in entrepreneurship: Introduction to the special issue. Industry and Higher Education, 30(3), 171-182. https://doi.org/10.1177/0950422216656699

Damásio, M. J., \&Bicacro, J. (2017). Entrepreneurship education for film and media arts: How can we teach entrepreneurship to students in the creative disciplines? Industry and Higher Education, 31(4), 253-266. https://doi.org/10.1177/0950422217713110

García-Cabrera, A., Déniz-Déniz, M., \&Cuéllar-Molina, D. (2016).Inteligencia emocional y emprendimiento: posibles líneas de trabajo. [Emotional intelligence and entrepreneurship: possible enquiry lines]. Cuadernos De Administración, 28(51), 65-101. https://doi.org/10.11144/Javeriana.cao28-51.ieep

Gilboy, M; Heinerichs , S., \&Pazzaglia, G. (2015). Enhancing Student Engagement Using the Flipped Classroom. Journal of Nutrition Education and Behavior, Volume 47, Issue 1, 109 - 114. https://doi.org/10.1016/.j.jneb.2014.08.008 
Galindo-Domínguez, H. (2018). Un meta-análisis de la metodología Flipped Classroom en el aula de Educación Primaria. [A meta-analysis of the Flipped Classroom methodology in the Primary Education classroom].Edutec. Revista Electrónica De Tecnología Educativa, (63), 73-85

(383). https://doi.org/10.21556/edutec.2018.63.983

Hassi, A. (2016). Effectiveness of early entrepreneurship education at the primary school level: Evidence from a field research in Morocco. Citizenship, Social and Economics Education, 15(2), 83-103. https://doi.org/10.1177/2047173416650448

llonen, S., \&Heinonen, J. (2018). Understanding affective learning outcomes in entrepreneurship education. Industry and Higher Education, 32(6), 391-404. https://doi.org/10.1177/0950422218805177

Kazakeviciute, A., Urbone, R., \&Petraite, M. (2016). Curriculum development for technology-based entrepreneurship education: A cross-disciplinary and crosscultural approach. Industry and Higher Education, 30(3), 202-214. https://doi.org/10.1177/0950422216656050

Landa-Cavazos, M. R., \&Ramírez Sánchez, M. Y. (2018). Diseño de un cuestionario de satisfacción de estudiantes para un curso de nivel profesional bajo el modelo de aprendizaje invertido. [Design of a student satisfaction questionnaire for a professional level course under the flipped classroom model]. Páginas De Educación, 11(2), 153-175. https://doi.org/10.22235/pe.v11i2.1632

Låg, T., \&Saele, R. G. (2019). Does the Flipped Classroom Improve Student Learning and Satisfaction? A Systematic Review and Meta-Analysis. AERA Open. https://doi.org/10.1177/2332858419870489

Lyons, M., Limniou, M., Schermbrucker, I., Hands, C., \&Downes, J. J. (2017). The Big Five, Learning Goals, Exam Preparedness, and Preference for Flipped Classroom Teaching: Evidence from a Large Psychology Undergraduate Cohort. Psychology Learning \& Teaching, 16(1), 36-46. https://doi.org/10.1177/1475725716680460

Madrid-García, E, Angulo-Armenta, J, Prieto-Méndez, M, Fernández-Nistal, M, \& Olivares-Carmona, K. (2018). Implementación de aula invertida en un curso propedéutico de habilidad matemática en bachillerato. [Implementation of Flipped Classroom in a propaedeutic course of mathematical skill in high school]. Apertura (Guadalajara, Jal.), 10(1), 24-39. https://dx.doi.org/10.32870/ap.v10n1.1149 
Mero-Chávez, E., Fernando-Pazmiño, M., \& San-Andrés, E. (2019). El flipped classroom como herramienta innovadora para el desarrollo del aprendizaje significativo.[The flipped classroom as an innovative tool for the development of meaningful learning].Cienciamatria, 6(10), 646-661. https://doi.org/10.35381/cm.v6i10.281

Miranda, A, \& Alfredo, M. (2018). Políticas y leyes de primer empleo en América Latina: tensiones entre inserción y construcción de trayectorias. [Policies and laws on youth employment in Latin America: tensions between job integration and trajectories building]. Revista de Ciencias Sociales, 31(42), 79-106. Recuperado de https://n9.cl/8pzf

Mitra, J. (2017). Holistic experimentation for emergence: A creative approach to postgraduate entrepreneurship education and training. Industry and Higher Education, 31(1), 34-50. https://doi.org/10.1177/0950422216684072

Muñoz-Morales, N., Barrientos-Oradini, N., Araya-Castillo, L., \& Reyes-Saavedra, J. (2019).Capacidades metacognitivas en el sistema educativo en instituciones educativas de educación media. [Metacognitive capacities in the educational system of educational institutions at secondary education].Revista Arbitrada Interdisciplinaria Koinonía, 4(7), 103-127. http://dx.doi.org/10.35381/r.k.v4i7.196

Osorio-Tinoco, F., \& Pereira-Laverde, F. (2011). Hacia un modelo de educación para el emprendimiento: una mirada desde la teoría social cognitiva. [Towards a model of education for entrepreneurship from the standpoint of cognitive social theory].Cuadernos De Administración, 24(43).

Osorio, F., \& Londoño-Roldán, J. C. (2016). Intención emprendedora de estudiantes de secundaria y preparatoria: usando el efecto de la exposición para extender la teoría de comportamiento planificado. [Entrepreneurial intention in middle and high school students: Using the exposure effect to extend the theory of planned behaviour]. Cuadernos De Administración, 28(51), 103-131. https://doi.org/10.11144/Javeriana.cao28-51.ieee

Oliver, A, Galiana, L, \& Gutiérrez-Benet, M. (2016). Diagnóstico y políticas de promoción del emprendimiento en estudiantes. [Assessment and promotion policies of entrepreneurship in students].Anales de Psicología, 32(1), 183189. https://dx.doi.org/10.6018/analesps.32.1.186681

Palacio-Vargas, C. (2015). La espiritualidad como medio de desarrollo humano. [Spirituality as a Means for Human Development].Cuestiones Teológicas, 42 (98), 459-481. 
Pierre-Álvarez R, \& Harris P. (2020). COVID-19 en América Latina: Retos y oportunidades. [COVID-19 in Latin America: Challenges and opportunities]. Revistachilena de pediatría, 91(2), 179-182.

Páez, A. (2018). Docentes y padres en el proceso de aprendizaje de los estudiantes. [Teachers and parents in the student learning process]. EPISTEME KOINONIA, 1(2), 18-34. http://dx.doi.org/10.35381/e.k.v1i2.509

Peche-Cruz, H., \& Giraldo-Supo, V. (2019). El Aprendizaje Flip Learning centrado en el estudiante como generador de calidad educativa. [Flip Learning focused on the student as a generator of educational quality]. Revista Arbitrada Interdisciplinaria Koinonía, 4(8), 427-450. http://dx.doi.org/10.35381/r.k.v4i8.293

Pulido-Acosta, F. \& Herrera-Clavero, F. (2019). Prediciendo el rendimiento académico infantil a través de la inteligencia emocional.[Predicting children's academic performance through emotional intelligence].Psicología Educativa, 25, 23-30. https://doi.org/10.5093/psed2018a16

Rodriguez, S., \&Lieber, H. (2020). Relationship Between Entrepreneurship Education, Entrepreneurial Mindset, and Career Readiness in Secondary Students. Journal of Experiential Education. https://doi.org/10.1177/1053825920919462

Rojas, G, Pertuz, V, Navarro, A, \& Quintero, L. (2019).Instrumento para Identificar Características Personales y Didáctica Utilizadas por los Docentes en la Formación de Emprendedores. [Instrument to Identify Personal and Didactic Characteristics Used by Teachers in the Training of Entrepreneurs]. Formación universitaria, 12(2), 29-40. https://dx.doi.org/10.4067/S0718-50062019000200029

Sánchez-García, J, Ward, A, Hernández, B, \&Flórez, J. (2017). Educación emprendedora: Estado del arte. [Entrepreneurial Education: State of the Art]. Propósitos $y \quad$ Representaciones, 5(2), 401473. https://dx.doi.org/10.20511/pyr2017.v5n2.190

Samuel, M. L. (2019). Flipped pedagogy and student evaluations of teaching. Active Learning in Higher Education. https://doi.org/10.1177/1469787419855188 
María Fernanda Argandoña-Mendoza; Carlos Enrique García-Vera; Patricio Alfredo Vallejo-Valdivieso

Uriguen-Aguirre, P, Pizarro-Romero, J, \&Cedeño-Flores, J. (2018).Metodologías de emprendimiento usadas en la Universidad Ecuatoriana: el caso de una institución de Educación Superior Orense. [Entrepreneurial methodologies used in the Ecuadorian University: The case of an Institution of Orense Higher Education].Revista Universidad y Sociedad, 10(3), 309-315.

Vidal-Ledo, M, Rivera-Michelena, N, Nolla-Cao, N, Morales-Suárez, I, \&Vialart-Vidal, M. (2016). Aula invertida, nuevaestrategiadidáctica. [The flipped classroom, a new didactic strategy]. EducaciónMédica Superior, 30(3), 678-688.

Yoon, S., Kim, S., \& Kang, M. (2018). Predictive power of grit, professor support for autonomy and learning engagement on perceived achievement within the context of a flipped classroom. Active Learning in HigherEducation. https://doi.org/10.1177/1469787418762463 